\title{
Un lieu où trouver du soutien
}

Daniel Cano

"Je suis un bachelier de 22 ans qui devait commencer son master à l'automne. Je suis depuis bientôt deux mois en confinement avec mes parents. Je perds la raison. [...] Ces derniers temps, tout cela me tire vers le bas. Toutes ces nouvelles de gens qui n'ont jamais de fière et qui soudain meurent [...]. Je pourrais en fait aussi tout simplement mourir. Je ne supporte pas l'idée que je pourrais éventuellement contaminer mes parents. Et si la situation est si grave, n'est-ce en ce cas pas inévitable que je tombe malade? Pourquoi devrais-je accumuler davantage de dettes pour ma formation si nul ne sait si je pourrai trouver un emploi, serai en mesure de payer mon loyer, trouverai une compagne?» (Source : Reddit)

C'est un post parmi des centaines que l'on peut lire sur un forum anglophone sur Reddit (reddit.com). Le forum sappelle «A place to come for support », Un lieu où trouver du soutien, qui propose pendant la crise du coronavirus une plateforme sur laquelle des gens peuvent séchanger sur leurs craintes et leurs soucis. Il en existe actuellement un nombre particulièrement élevé. L'incertitude, des peurs existentielles et le manque de proximité humaine pèsent sur le moral. C’est surtout ce dernier point qui pose problème, parce qu'il n'existe aucun remède à ce niveau, rapporte Veronica Defièbre, vice-présidente de l'Association suisse des psychothérapeutes, dans une interview : "Mais ce qui d’après moi pèse beaucoup à la plupart des gens, c'est la longue durée de l'interdiction de contact corporel lorsqu'ils vivent seuls, et l'incertitude quant au moment où ils pourront à nouveau rencontrer d'autres personnes et toucher ces dernières - ne fût-ce que pour simplement leur serrer les mains ou les prendre brièvement dans leurs bras. » Allier le télétravail et la famille sous un même toit fait également partie des défis à relever actuellement. Certains de ces défis sont, pour les gens souffrant de pathologies psychiques, également une réalité quotidienne en dehors de la pandémie.

\section{Peur de l'incertitude}

" J'entends que la plupart des gens pourraient éventuellement retourner à la normalité, pendant que les groupes à risque devraient continuer à s'isoler. En tant que membre d'un groupe à risque, cela me déprime extrêmement. Ce confinement n'a jamais été simple, toujours est-il que nous étions tous embarqués dans le même bateau. La pensée que d'autres pourraient renouer avec leur ancienne vie pendant que je resterais prisonnier en isolement, me fait complètement retomber en dépression. »(Source: Reddit)

Defièbre explique : « Les gens souffrant de pathologies psychiques ont souvent des difficultés à faire face à la situation dêtre livrés à eux-mêmes. Ils ne parviennent plus à faire le tri dans leurs idées, se concentrent sur leurs problèmes psychiques et tournent alors souvent en rond. » L'isolement peut tout à fait renforcer de tels symptômes. On peut également penser que le nombre de gens souffrant de pathologies psychiques aura augmenté après la pandémie. Cela dépend cependant de plusieurs facteurs : quand précisément retournerons-nous à la situation "d’avant le coronavirus " ? Y aura$\mathrm{t}$-il de nouvelles vagues de contamination ? Cest avant tout la capacité de léconomie à surmonter la crise qui sera décisive. Les gens qui doivent maintenant fermer leurs entreprises ou qui perdent leur emploi en souffriront psychiquement. Defièbre se fait des soucis à propos des victimes d'agressions domestiques : " Si une hausse de la violence et des agressions sexuelles se produit effectivement au sein des familles, comme semblent le dire des statistiques allemandes, il en résultera sûrement des dommages à long terme, qui entraîneront des thérapies et des séjours en clinique pendant des années. » La rapidité avec laquelle les personnes concernées pourront surmonter cette crise dépend de nombreux facteurs - mais notamment des capacités d'adaptation psychiques et de la qualité de laccompagnement psychothérapeutique des personnes concernées, dit Defièbre : " Il se peut également que notre perception de nous-mêmes et de notre impression de tout avoir en mains sera durablement changée et affaiblie par cette pandémie. »

\section{Toutes les thérapies \\ ne sont pas identiques}

Il est également difficile dévaluer les ressources disponibles pour aider ces gens. Defièbre veut dire que : "D'un point de vue purement statistique, cela veut toujours dire qu'il y aurait suffisamment de psychothérapeutes, tout au moins dans les agglomérations urbaines. Mais d’après mon expérience, les patientes et les patients, même dans des villes telles que Zurich, doivent souvent attendre plusieurs mois pour obtenir un rendez-vous de thérapie. Je connais de nombreux collègues qui ne prennent absolument plus

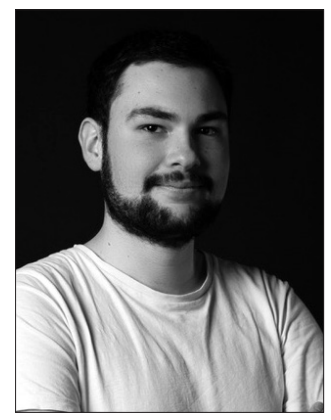




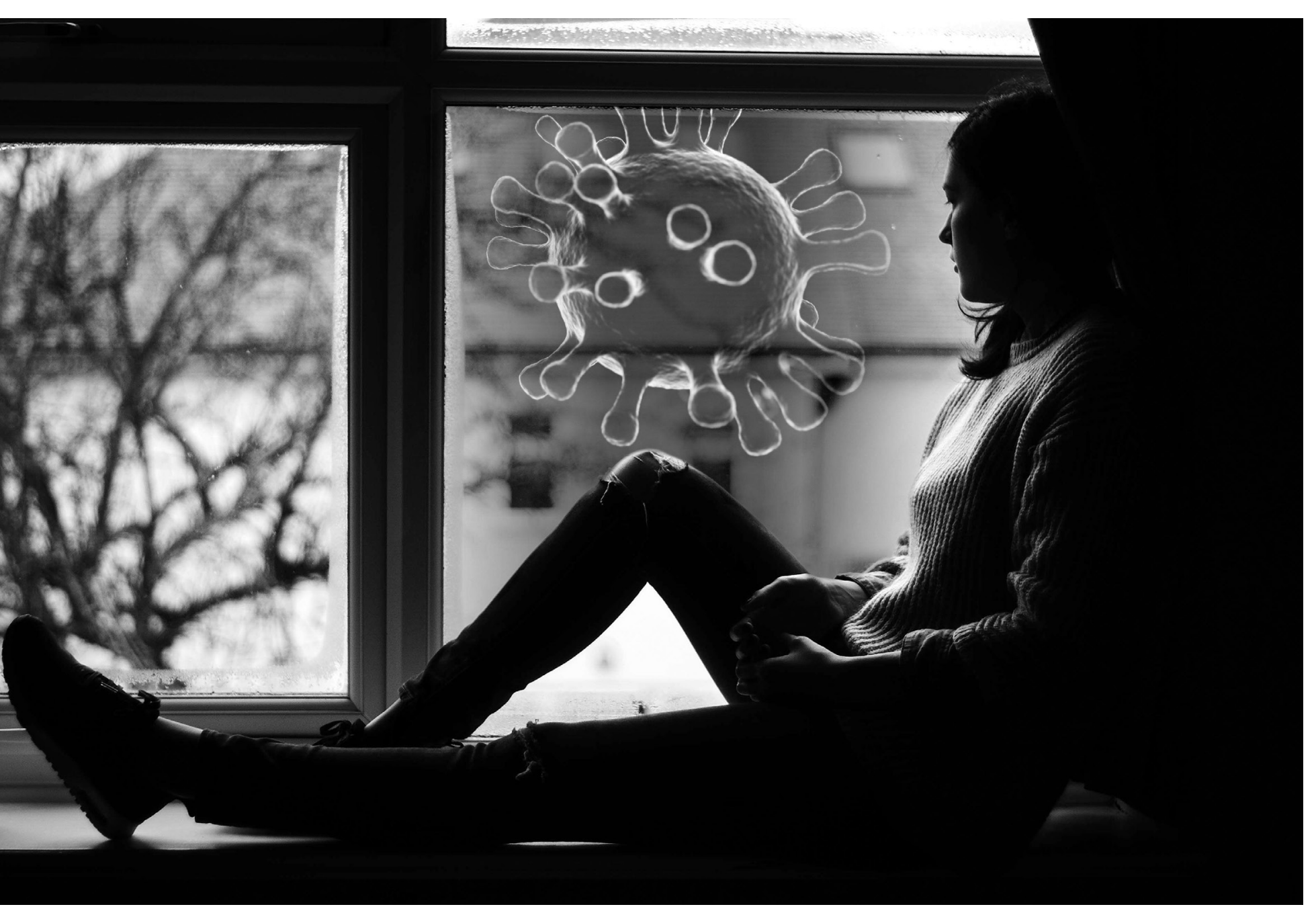

de nouvelles patientes et de nouveaux patients pendant certaines périodes. Je ne pense donc pas que nous ayons suffisamment de thérapeutes si la demande continue encore à augmenter. " La psychothérapeute Susanne Heule pense que le problème se trouve ailleurs. Elle affirme dans l'interview : " Il y aurait bien suffisamment de thérapeutes si la psychothérapie psychologique était enfin traitée de la même manière que les thérapies médicales en étant ainsi remboursable par l'assurance de base. Il s'agit également de faire en sorte qu'il y ait suffisamment de places de thérapie finançables pour les patientes et les patients. » Elle aborde ici un point de conflit persistant depuis de nombreuses années, car les prestations de psychothérapeutes ne peuvent être remboursées par la caisse d'assurance maladie que si elles sont décomptées via un ou une psychiatre. Cette inégalité apparaît également ailleurs : les psychothérapeutes peuvent désormais - tout au moins pendant la crise actuelle - décompter jusquà 360 au lieu de 240 minutes de thérapie téléphonique par semestre par le passé. Une telle limitation n'existe pas pour les psychiatres. ${ }^{1}$

La crise du coronavirus ne devrait, selon Defièbre, pas mener actuellement à la percée de méthodes de thérapie numériques. Lobstacle technique devrait rester trop grand pour les patientes et les patients âgés, et beaucoup d’entre eux préfèreraient la salle de thérapie protégée à

1 Source: fiche d'information de l'OFSP relative à la prise en charge des coûts pour les prestations ambulatoires à distance pendant la pandémie de COVID-19 du 2 avril 2020. 
leur propre appartement, explique-t-elle. Pour elle aussi, en tant que thérapeute, le contact personnel représenterait une aide précieuse : «Il est impossible d'enregistrer les vibrations des sentiments par vidéo comme on peut le faire lorsqu'un patient/une patiente est assis(e) devant vous dans votre cabinet. " Mais Defièbre voit aussi des opportunités au-delà des obstacles : «Cela reste une bonne possibilité de proposer tout de même aux patientes et aux patients qui ne peuvent pas venir au cabinet, par exemple pour des raisons médicales, des rendez-vous de thérapie au cours desquels ils peuvent voir et parler au ou à la thérapeute. Mais la sécurité des données et la qualité de la transmission doivent être encore améliorées. »

Et comment se présentent les choses avec léchange dans des forums sur Internet évoqué tout au début ? « Pour moi, cette manière de procéder n'est pas très différente qu'un groupe de soutien sans direction thérapeutique. Cest quelque chose qui existe depuis longtemps et qui a été jugé la plupart du temps comme très profitable par les participants », constate Defièbre.

"J'ai les mêmes pensées et sentiments que vous. Je suis passé par une phase de dépression de plusieurs jours, javais alors ensuite quelques bonnes journées, puis voulais plus tard à nouveau simplement tout laisser tomber. La vérité est que nous sommes tous plongés dans la même affaire, certains se portant mieux, d'autres plus mal. Mais vos sentiments sont justifiés. [...] Il y aura à nouveau des choses dont nous pourrons nous réjouir, même s'il nén paraît pas. Je suis désolé que vous vous sentiez ainsi, mais jespère que cela vous aidera de savoir que vous nềtes pas tout seul. » (Source : Reddit)

\section{Discussions d'experts}

Interview par mail (le 3 mai 2020) avec Veronica Defièbre, vice-présidente de l'Association suisse des psychothérapeutes, directrice de la Conférence suisse de la Charte, psychothérapeute reconnue par la confédération.

Interview par mail (2 mai 2020) avec Susanne Heule, psychothérapeute reconnue par la Confédération, thérapeute naturelle intégrative.

Daniel Cano est étudiant en production multimédia à la Haute école spécialisée des Grisons. Outre son travail en tant que producteur vidéo, il est responsable de la production de la série Web Schuldig (Coupable). En privé, il s'occupe volontiers de l'interface entre la société et la technologie ainsi que de leurs opportunités et leurs risques.

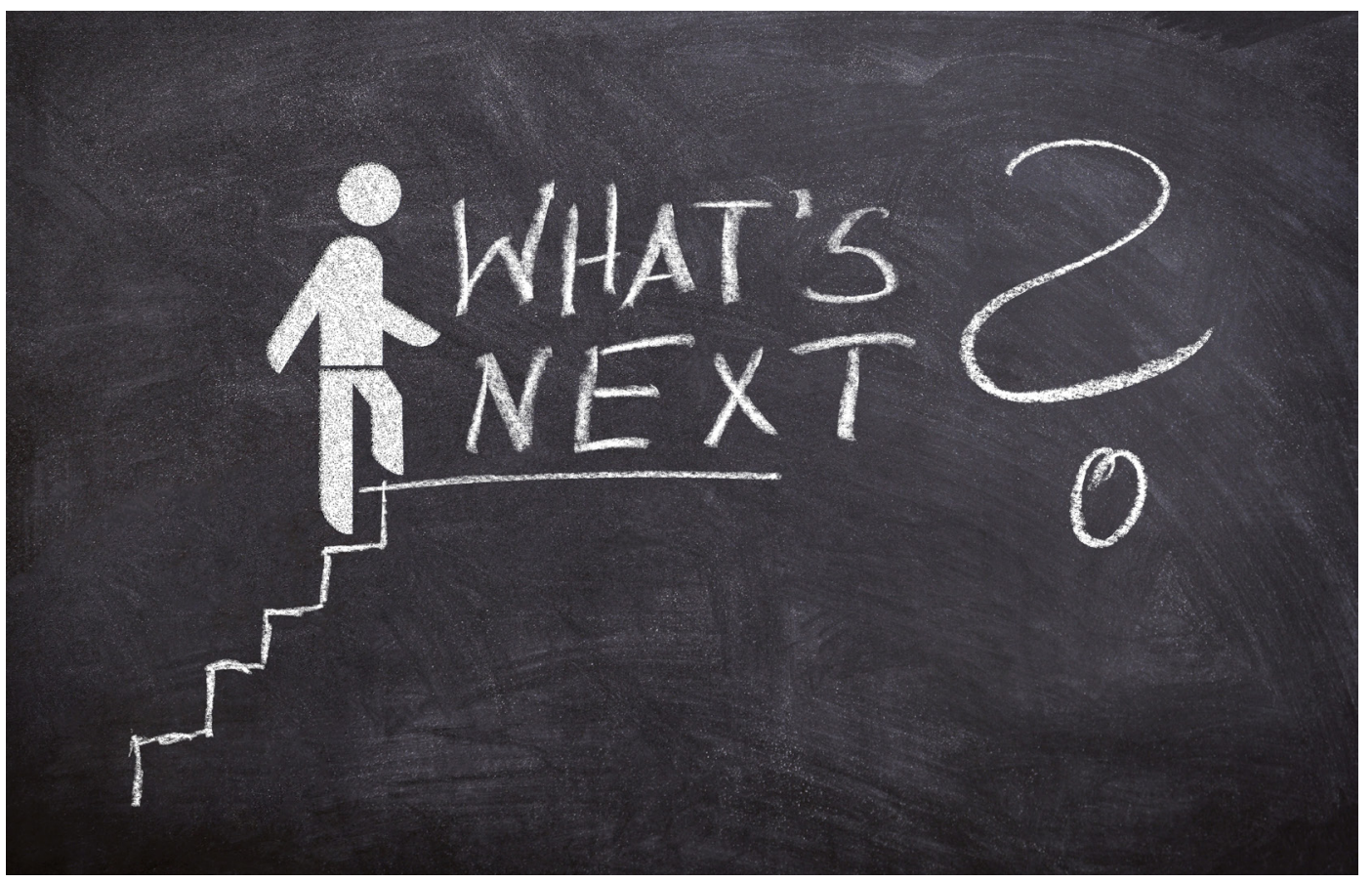

